\title{
Prognostic utility of baseline neutrophil-to- lymphocyte ratio in patients receiving immune checkpoint inhibitors: a review and meta-analysis
}

This article was published in the following Dove Press journal:

OncoTargets and Therapy

\author{
Danielle Benedict Sacdalan' \\ Josephine Anne Lucero² \\ Dennis Lee Sacdalan' \\ 'Section of Medical Oncology, \\ Department of Medicine, University \\ of the Philippines Manila and \\ Philippine General Hospital, Manila, \\ Philippines; 'Department of Medicine, \\ University of the Philippines Manila \\ and Philippine General Hospital, \\ Manila, Philippines
}

Introduction: Systemic inflammation is associated with prognosis in solid tumors. The neutrophil-to-lymphocyte ratio (NLR) is a marker for the general immune response to various stress stimuli. Studies have shown correlation of NLR to outcomes in immune checkpoint blockade, peripheral neutrophil count to intratumor neutrophil population, and NLR to intratumoral levels of myeloid-derived suppressor cells. Studies have shown elevated peripheral blood regulator T cells accompanied by elevated NLR are associated with poor outcomes further highlighting the importance of inflammation in the prognosis of cancer patients.

Methods: We performed a meta-analysis of published articles on the utility of baseline NLR in predicting outcomes in patients treated with immune checkpoint inhibitors (ICIs) using Review Manager, version 5.3. Seven studies on the prognostic utility of NLR in ICI treatment were included in this analysis. For outcomes of interest, the hazard ratios (HRs) were computed. Subgroup analyses were planned based on type of malignancy and type of immune checkpoint inhibitor.

Results/discussion: A high NLR resulted in worse overall survival (OS) (HR, 1.92; 95\% CI, $1.29-2.87 ; p=0.001$ ) and progression-free survival (PFS; HR, 1.66; 95\% CI, 1.38-2.01; $p<0.00001$ ) across types of malignancies studied (melanoma, non-small-cell lung cancer, and genitourinary cancer). Subgroup analysis across different types of malignancies treated with ICI showed similar results for OS and PFS. The single study on genitourinary cancers also showed worse OS and PFS (OS: HR, 1.82; 95\% CI, 1.29-2.87; $p=0.001$ and PFS: HR, 1.83; 95\% CI, 0.97-3.44; $p=0.06$ ). A high NLR also showed worse OS and PFS across all ICIs (ipilimumab, nivolumab, and unspecified or pooled pembrolizumab and nivolumab; OS: HR, 1.92; 95\% CI, 1.29-2.87; $p=0.001$ and PFS: HR, 1.66; 95\% CI, $1.38-2.01 ; p<0.00001)$. Subgroup analysis by type of ICI showed similar results.

Conclusion: A high NLR is associated with poorer outcomes across studies. This shows that NLR has the potential as a readily available prognostic indicator for patients receiving ICI based on available studies. Studies utilizing more stringent design may serve to better determine the utility of this tool.

Keywords: neutrophil-to-lymphocyte ratio, immunotherapy, biomarkers, inflammation

\section{Introduction}

An increasing interest has followed immunologic checkpoint blockade beginning with the approval of the first agent, ipilimumab. As studies continue to show notable and durable response to checkpoint inhibition across different indications, the search for biomarkers that will predict response to therapy has gained pace. In addition, the role of inflammation in cancer immunology and its effect on cancer immunotherapy merits scrutiny. Studies have shown consistently that inflammation is associated with 
prognosis in solid tumors due to its effect on the immune response to the disease. . $3,8,9,13,17,21,23-25$ Specifically, the association between inflammation and immunosuppression, which is one of the hallmarks of cancer, is pointed out. ${ }^{17,21,45}$ In this context, different biomarkers have been investigated in predicting response to immune checkpoint blockade apart from programmed cell death protein 1 (PD-1)/programmed death ligand 1 (PD-L1) and cytotoxic T-lymphocyte-associated antigen 4 (CTLA-4). These biomarkers include professional immunoregulatory cells, soluble mediators such as adenosine, and neutrophil-to-lymphocyte ratio (NLR). ${ }^{8,18}$

\section{CTLA-4 and PD-I/PD-LI}

The regulation of effector $\mathrm{T}$-cell function underpins the host immune response to self- versus non-self-antigens. Binding of the T-cell receptor (TCR) to a major histocompatibility complex (MHC)-bound antigen on an antigen-presenting cell (APC) is the determinant of its subsequent fate. $T$ cells that bind to self-antigens and those that display insufficient affinity for MHC are eliminated in the thymus. Meanwhile, $\mathrm{T}$ cells that show weak affinity for MHC-bound antigens (including self) are released into the blood and lymphoid organs as naive T cells. In these peripheral sites, naive T cells are exposed to professional APCs that provide the costimulatory signal that activates them. Here, the role of immune checkpoint pathways mediated by CTLA-4 and PD-1 plays a central role in immunoregulation by inhibiting the activity of T cells with an affinity for self-antigens. ${ }^{5}$

Although binding of the TCR to MHC-bound antigen confers specificity to the $\mathrm{T}$ cell, its activation will require further costimulatory signals. Binding of B7 ligand on APCs to $\mathrm{CD} 28$ receptor on $\mathrm{T}$ cells leads to signaling within the $\mathrm{T}$ cell that results in its proliferation, differentiation, and survival. Regulation of this process is achieved through the binding of another receptor, CTLA-4 to B7. The amount of CD28:B7-to-CTLA-4:B7 binding governs whether the T cell will become activated or anergic. It is postulated that CTLA4:B7 binding induces inhibitory signals that oppose activating signals from TCR:MHC and CD28:B7 binding. 5,43,50

A similar result is seen in the binding of PD-1 on T cells with PD-L1/2 on APCs. PD-1 is a marker of T-cell exhaustion, which when activated results in downregulation of T-cell function. This process has been observed in disease states such as chronic infection and cancer. ${ }^{5,43}$

Both CTLA-4 and PDL-1 are members of the B7/CD28 superfamily of costimulatory receptors. Although the activation of both pathways induces negative effects on T-cell activity, they two differ in terms of timing of downregulation and the anatomic location where the activity of each receptor can be observed. The activity of CTLA-4 is observed during the priming phase of T-cell activation, whereas the activity of PD-1 occurs mainly during the effector phase. CTLA-4 binds to B7, which is found on APCs in the lymph nodes and the spleen. Meanwhile, PD-1 binds to PD-L1/2, which is more widely expressed, being found in lymphoid and non-lymphoid cells as well as in cancer cells. Therefore, its action is primarily seen in peripheral tissues. ${ }^{5,8,46,50}$

\section{Immune checkpoint inhibition and the challenge of predictive biomakers}

Currently, immune checkpoint inhibitors (ICIs) that target CTLA-4 or PD-1/PD-L1 signaling are used for the treatment of cancers. Solid tumors with approved indications for ICIs are metastatic melanoma, non-small-cell lung cancer (NSCLC), gastroesophageal or gastric cancer, hepatocellular carcinoma, microsatellite instability-high (MSI-H) colorectal cancer, head and neck squamous cell carcinoma, renal cell carcinoma ( $\mathrm{RCC}$ ), urothelial cell carcinoma, classical Hodgkin's lymphoma, and metastatic Merkel cell carcinoma. ${ }^{11,22,23,30,36}$

Pembrolizumab, a monoclonal antibody against PD-1, is one of the currently used ICIs. Its use in NSCLC is the only one guided by a biomarker - the tumor proportion score (TPS) of PD-L1 expression. In the first-line treatment of epidermal growth factor receptor (EGFR) and anaplastic lymphoma kinase (ALK) mutation-negative, NSCLC, the use of pembrolizumab is recommended if the tumor manifests a high PD-L1 expression defined as a TPS of at least $50 \%$. In the second-line treatment and beyond, a minimum TPS of $1 \%$ is required. In addition, if the tumor is EGFR or ALK mutation positive, then the progression needs to have followed the treatment with the appropriate targeted agent. For mutation-negative tumors, progression needs to have followed the treatment with a platinum-based regimen. ${ }^{5,36}$ In contrast, there are no recommended PD-L1 expression levels for nivolumab, which is another anti-PD-1 monoclonal antibody. ${ }^{30}$

The inability to estimate which patients will benefit from ipilimumab continues to be a source of consternation. CTLA-4 is an unreliable biomarker for response, and other markers that are possibly associated with outcomes in ipilimumab therapy such as absolute lymphocyte count, inducible costimulatory (ICOS) molecule, and NY-ESO-1 have been investigated; however, none of these have been validated. ${ }^{3,5}$

Due to the lack of reliability and generalizability of PD-L1 and CTLA-4, the possible utility of other biomarkers 
such as professional immunoregulatory cells, soluble mediators such as adenosine, and, particularly, the NLR also warrant investigation and review. ${ }^{8,18}$

\section{Neutrophils and inflammation}

Neutrophils exhibit heterogeneity of function in the tumor microenvironment, which can be categorized into two phenotypes - high-density neutrophils (HDNs) and low-density neutrophils (LDN). The HDN phenotype promotes antitumor effects either through direct action against tumor cells or by stimulating T-cell-mediated immunity. In contrast, the LDN phenotype favors tumor progression by promoting T-cell suppression through the expression of arginase and upregulating tumor angiogenesis through vascular endothelial growth factor (VEGF). Set against the backdrop of inflammation, neutrophils exhibit the HDN phenotype predominantly during the early phases of inflammation, whereas the LDN phenotype tends to accumulate when inflammation resolves. ${ }^{29,36,40}$

Cancer can be viewed as a chronic inflammatory process, wherein HDNs are generated overtime. Notably, tumor-associated inflammation does not completely resolve due to the concerted effects of genetically altered tumor cells, hypoxia, and cell death. As a result, LDN neutrophils accumulate overtime, leading to an overall unfavorable neutrophil phenotype in the tumor microenvironment. Moreover, the proportion of LDN tends to increase in relation to tumor burden. It is of interest to note that in murine models, exposure of HDN to transforming growth factor (TGF)- $\beta$ leads to a conversion to the LDN phenotype. TGF- $\beta$ functions both as an effector cytokine in the resolution of inflammation and as a promoter of epithelial-mesenchymal transition (EMT), leading to tumor progression and metastasis. ${ }^{29,38,40,42}$

\section{Professional immunoregulatory cells - myeloid-derived suppressor cells (MDSCs) and regulatory $T$ cells $\left(T_{\text {regs }}\right)$}

Granulocyte myeloid-derived suppressor cells (gMDSCs) are closely related to neutrophils. This term describes a subset of myeloid cells expressing neutrophilic markers, such as human CD15 and CD66b, accompanied by a suppressive phenotype..$^{15,33,55}$ These cells play an important role in tumor progression by the following mechanisms: 1) regulation of T-cell and natural killer (NK)-cell antitumor activity, 2) facilitation of tumor neovascularization, 3) assistance in tumor invasion of proximal and distal sites, and 4) enabling EMT. ${ }^{10,55}$

Effector T cells, particularly those of cytotoxic $\left(\mathrm{CD}^{+}\right)$ $\mathrm{T}$ cells, play a central role in the immune response to cancer; and the engagement of effector $\mathrm{T}$ cells and NK cells in the tumor microenvironment is the goal of successful immunotherapy. ${ }^{31,44}$ High-density infiltration of tumor by these cells has been associated with earlier tumor stage and a more favorable prognosis for patients. ${ }^{17,38}$ In contrast to cytotoxic $\mathrm{T}$ cells, $\mathrm{T}_{\text {regs }}$ most commonly defined by their expression of a nuclear transcription factor, FOXP3, are thought to attenuate the immune response to cancer by suppressing the activity of cytotoxic $\mathrm{T}$ cells via cell-tocell contact and the release of cytokines, notably TGF- $\beta$. Conversely, the reduction of $\mathrm{T}_{\text {regs }}$ in the peripheral blood of patients reinvigorates the immune response to tumor based on studies of colorectal cancer. ${ }^{5,38}$ However, recent studies have shown that $\mathrm{T}_{\text {reg }}$ infiltration of tumors portends a more favorable prognosis in specific cancer types such as follicular lymphoma, diffuse large B-cell lymphoma, Hodgkin's lymphoma, and even colorectal cancer, which possibly reflects the effect of the type of cancer involved and its respective microenvironment on the phenotype of the infiltrating $\mathrm{T}_{\text {reg. }}{ }^{16}$ In addition, various studies attribute this phenomenon to the heterogeneity of $\mathrm{T}_{\text {regs }}$, showing the need to more specifically describe a given population beyond FOXP3 positivity alone. ${ }^{1,15,39,47,48}$ Although the definite role of $\mathrm{T}_{\text {regs }}$ in the tumor microenvironment is unclear, the increased presence of $\mathrm{T}_{\text {regs }}$ and MDSC in human tumors as well as in the circulation of patients with cancer has been widely described. ${ }^{48}$

\section{Adenosine and tumor hypoxia}

Adenosine is a purine nucleoside, which affords a variety of functions depending on its action in the intracellular or extracellular compartment. Intracellularly, adenosine plays a role in cellular energetics, the methionine cycle, and the metabolism of nucleic acids. Meanwhile, extracellularly, it plays a role in immunoregulation. ${ }^{33,44}$ Extracellular adenosine exerts its effects by binding to one of the four G-proteincoupled adenosine receptors - A1R, A2AR, A2BR, and $\mathrm{A} 3 \mathrm{R}$. These receptors are subdivided based on their ability to induce downstream signaling via cAMP. Induction of cAMP signaling via A2AR and A2BR is associated with immunosuppression. In contrast, inhibition of cAMP signaling via $\mathrm{A} 1 \mathrm{R}$ and $\mathrm{A} 3 \mathrm{R}$ is associated with immunopromotion. ${ }^{45}$

The action of the ectonucleotidases CD39 and CD73 enables the conversion of ATP to adenosine, which leads to its accumulation in the tumor microenvironment particularly in the setting of hypoxia. Low oxygen levels in the tumor microenvironment caused by an erratic tumor blood supply, high metabolic demand by the tumor, and even inflammation force cells in the tumor microenvironment to switch from an 
aerobic to an anaerobic type of metabolism. This switch is facilitated by hypoxia-inducible factor- $1 \alpha$ (HIF-1 $\alpha)$. HIF- $1 \alpha$ also induces CD39, CD73, and A2BR expression, thus promoting adenosine-mediated immunosuppression. Notably, this same process has been reversed in mouse models by whole body exposure to a hyperoxic atmosphere. ${ }^{45}$ Also, in murine studies, HIF-1 $\alpha$ has also been found to increase tumor infiltration by FOXP3 $3^{+} \mathrm{T}_{\text {reg }}{ }^{34,45}$

\section{NLR}

Systemic inflammation is associated with prognosis in solid tumors. ${ }^{2,4,27,37,49,52-54}$ NLR is a marker for the general immune response to various stress stimuli. ${ }^{18,20,37}$ Studies have also correlated the NLR with other predictive biomarkers of outcomes in immune checkpoint blockade. The peripheral neutrophil count measured by the NLR has been found to be directly correlated with the intratumor neutrophil population. ${ }^{29}$ Studies have reported a direct correlation between NLR and intratumoral levels of gMDSCs. ${ }^{19,33}$ Finally, elevated levels of peripheral blood $\mathrm{T}_{\text {regs }}$ accompanied by an elevated NLR were associated with a poor prognosis for patients with pancreatic cancer. ${ }^{8}$

\section{Research question}

In patients with cancer receiving ICI therapy, what is the utility of the NLR in predicting overall survival (OS) and progression-free survival (PFS)?

\section{Methods}

\section{Search strategy and study selection}

A systematic review was conducted to identify studies on the relationship of the NLR with OS and PFS in patients treated with ICIs. A search for relevant published and unpublished studies was performed using PubMed, ScienceDirect, Google Scholar, and the American Society of Clinical Oncology (ASCO) meetings library. The search terms utilized were NLR, immune checkpoint inhibitor response, prognosis, nivolumab, pembrolizumab, and ipilimumab. The last search was updated on June 24, 2017. Both free text and medical subheadings (MeSH) terms were used in the search strategy.

Included studies involved 1) human subjects receiving immune checkpoint inhibitor therapy for solid tumors and 2) determination of the relationship between baseline NLR and prognosis (OS and PFS).

Exclusion criteria were 1) absent baseline NLR values; 2) concomitant use of a chemotherapeutic agent with the ICI; 3) analysis of NLR in combination with other patient variables (eg, NLR + lactate dehydrogenase [LDH]);
4) reviews, letters, commentaries, case reports, expert opinions, and nonhuman studies; 5) incomplete or non-analyzable data; and 6) publication in a language other than English, which did not have an English translation.

\section{Data extraction}

All searches were conducted independently by the first two investigators. They independently extracted data on the authorship and publication history of the studies included. Data on cancer studied, NLR cutoffs, type of ICIs investigated, and the outcome measures used (hazard ratio [HR] with $95 \% \mathrm{CI}$ ) were also obtained from the included studies. Any discrepancy was resolved by consensus and in consultation with the third author.

Jeyakumar et $\mathrm{a}^{24,25}$ presented two reports with OS and PFS data for melanoma combined with genitourinary (GU) cancers $^{24}$ (RCC and urothelial carcinoma [UC]), respectively. Both papers were presented as abstracts at the 2017 session of ASCO. Due to uncertainties regarding overlap for the data set on GU cancer, the authors of this meta-analysis decided to use only the data on melanoma from the first study and the data on GU cancers from the second study. ${ }^{25}$ No data for the ICI pembrolizumab were obtained, but a study ${ }^{41}$ that looked at patients receiving anti-PD-1 therapy (pembrolizumab or nivolumab) was included in this analysis.

\section{Statistical analysis}

Analysis of the relationship between NLR and prognosis in ICI therapy was performed according to the cancer site (melanoma, lung, and GU cancers) and the type of ICI used (ipilimumab, nivolumab, unspecified, or pooled). HRs with their 95\% CIs from each study were used to calculate pooled HRs. Testing of heterogeneity of pooled results was performed using Cochran's Q test and Higgins $I^{2}$ statistic. A value of $p<0.10$ for $\mathrm{Q}$ test was considered statistically significant, and the random-effects (DerSimonian-Laird method) model was applied to calculate the pooled HRs. ${ }^{6}$ Publication bias of literatures was evaluated using Begg's funnel plot and Egger's linear regression test, and a value of $p<0.05$ was considered statistically significant. All statistical analyses were performed using Review Manager, version 5.3 (The Nordic Cochrane Centre, Copenhagen, Denmark).

\section{Results \\ Study characteristics}

A total of seven articles were obtained using the inclusion and exclusion criteria outlined earlier. Figure 1 details the data extraction process undertaken by the authors. All included 


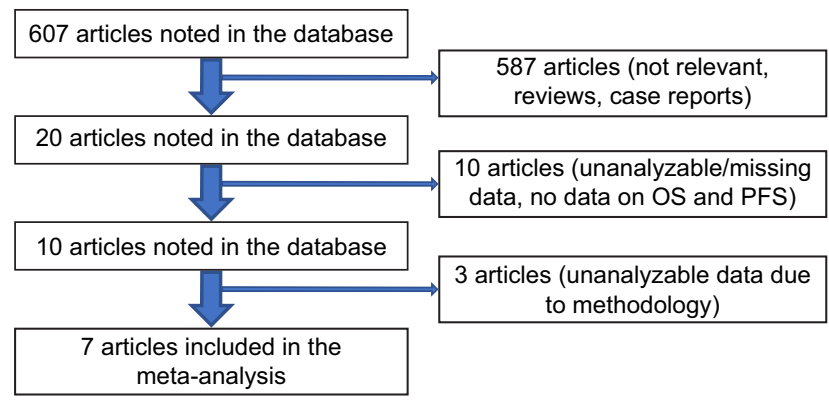

Figure I Flowchart of search strategy for meta-analysis on prognostic utility of the NLR in patients receiving immune checkpoint inhibitors.

Abbreviations: NLR, neutrophil-to-lymphocyte ratio; OS, overall survival; PFS, progression-free survival.

studies were published in English. Three studies were on melanoma, three studies were on NSCLC, one study was on GU cancers, and one study was on both melanoma and GU cancer (see Methods for how this was handled). Of the seven included studies, six were carried out in the United
States and one was carried out in France. Only three studies reported on the ethnicity of their participants. Bagley et $\mathrm{al}^{2}$ described participants as Caucasian, African American, Asian, or others. Meanwhile, Jeyakumar et al ${ }^{24,25}$ described their participants as African American or other. Regarding the type of ICI used, two studies reported on ipilimumab, ${ }^{7,55}$ two studies reported on nivolumab, ${ }^{2,35}$ one study reported on pooled data from nivolumab and pembrolizumab, ${ }^{41}$ and the remaining two studies did not specify the type of ICI used. ${ }^{24,25}$ Table 1 summarizes the characteristics of included studies.

\section{Risk of bias}

Figure 2 summarizes the data for risk bias across included studies. All studies included were cohort studies. Five of the seven studies were of retrospective design that utilized registries to obtain patient data. None of the included studies reported on co-interventions given (eg, colony-stimulating factors for supportive care). Cancer stage and Eastern

Table I Characteristics of included studies for the meta-analysis on prognostic utility of the NLR in patients receiving immune checkpoint inhibitors

\begin{tabular}{|c|c|c|c|c|c|c|c|}
\hline Characteristics & $\begin{array}{l}\text { Cassidy et } \mathrm{al}^{7} \\
(2017)\end{array}$ & $\begin{array}{l}\text { Zaragoza et } \mathrm{al}^{55} \\
(20 \mid 6)\end{array}$ & $\begin{array}{l}\text { Bagley et } \mathrm{al}^{2} \\
(2017)\end{array}$ & $\begin{array}{l}\text { Patil et al }{ }^{35} \\
(2017)\end{array}$ & $\begin{array}{l}\text { Soyano et } \mathrm{al}^{41} \\
(20 \mid 7)\end{array}$ & $\begin{array}{l}\text { Jeyakumar } \\
\text { et } \mathrm{al}^{24} \\
(\mathrm{melanoma}) \\
(20 \mid 7)\end{array}$ & $\begin{array}{l}\text { Jeyakumar et } \mathrm{al}^{25} \\
\text { (GU) (20I7) }\end{array}$ \\
\hline Population & $\begin{array}{l}\text { I } 97 \text { Stage III and } \\
\text { IV melanoma } \\
\text { treated from } \\
2006 \text { to } 201 \text { I }\end{array}$ & $\begin{array}{l}58 \text { Stage III and IV } \\
\text { unresectable } \\
\text { melanoma } \\
\text { treated from } \\
2008 \text { to } 2014\end{array}$ & $\begin{array}{l}175 \text { advanced } \\
\text { NSCLC treated } \\
\text { outside a clinical } \\
\text { trial from } \\
2015 \text { to } 2016\end{array}$ & $\begin{array}{l}\text { I I5 NSCLC } \\
\text { treated with } \\
\text { nivolumab } \\
\text { treated } \\
\text { in a single } \\
\text { institution }\end{array}$ & $\begin{array}{l}52 \text { patients with } \\
\text { advanced NSCLC } \\
\text { treated in a single } \\
\text { institution }\end{array}$ & $\begin{array}{l}84 \text { patients } \\
\text { with melanoma } \\
\text { treated with } \\
\text { immune } \\
\text { checkpoint } \\
\text { inhibitors }\end{array}$ & $\begin{array}{l}57 \text { patients with } \\
\text { advanced GU } \\
\text { cancer (RCC } \\
\text { and UC) }\end{array}$ \\
\hline $\mathrm{ICl}$ & $\begin{array}{l}\text { Ipilimumab } \\
\text { ( } 3 \text { or } 10 \mathrm{mg} / \mathrm{kg} \\
\text { q3 weeks for up } \\
\text { to four doses) }\end{array}$ & $\begin{array}{l}\text { Ipilimumab } \\
\text { ( } 3 \text { or } 10 \mathrm{mg} / \mathrm{kg} \\
\text { q3 weeks for up } \\
\text { to four doses } \\
\text { followed by } \\
\text { maintenance) }\end{array}$ & $\begin{array}{l}\text { Nivolumab } \\
(3 \mathrm{mg} / \mathrm{kg} \text { every } \\
2 \text { weeks without } \\
\text { concomitant } \\
\text { treatment) }\end{array}$ & Nivolumab & $\begin{array}{l}\text { Nivolumab }(\mathrm{N}=48) \text {; } \\
\text { pembrolizumab } \\
(\mathrm{N}=4)\end{array}$ & $\begin{array}{l}\mathrm{ICl} \text { not } \\
\text { specified }\end{array}$ & $\mathrm{ICl}$ not specified \\
\hline NLR calculation & $\begin{array}{l}\text { NLR calculated } \\
\text { at baseline and } \\
\text { q3 weeks until } \\
9 \text { weeks }\end{array}$ & $\begin{array}{l}\text { NLR measured } \\
\text { continuously, at } \\
\text { weeks I and } 7\end{array}$ & $\begin{array}{l}\text { NLR taken at } \\
\text { baseline }\end{array}$ & $\begin{array}{l}\text { NLR taken } \\
\text { at baseline } \\
\text { and after } \\
\text { two cycles }\end{array}$ & $\begin{array}{l}\text { NLR taken at } \\
\text { baseline }\end{array}$ & $\begin{array}{l}\text { NLR taken } \\
\text { at baseline } \\
\text { and after four } \\
\text { doses of } \mathrm{ICI}\end{array}$ & $\begin{array}{l}\text { NLR taken at } \\
\text { baseline }\end{array}$ \\
\hline NLR cutoff & $\geq 5$ and $<5$ & $\geq 4$ and $<4$ & $\geq 5$ and $<5$ & $\geq 2.8$ or $<2.8$ & $>4.59$ or $\leq 4.59$ & $N L R \geq 4$ or $<4$ & NLR $\geq 4$ or $<4$ \\
\hline Median follow-up & 54.3 months & 931 days & Not stated & Not stated & I3.6 months & Not stated & Not stated \\
\hline $\begin{array}{l}\text { Outcome of } \\
\text { interest }\end{array}$ & OS and PFS & OS & OS and PFS & OS & OS and PFS & OS and PFS & OS and PFS \\
\hline Results & $\begin{array}{l}\text { For NLR } \geq 5 \text { : } \\
\text { HR for death, } \\
2.03 \text { ( } 95 \% \mathrm{Cl} \text {, } \\
\text { I.49-2.77); HR } \\
\text { for progression, } \\
\text { I.8I (95\% Cl, } \\
\text { I.33-2.45) }\end{array}$ & $\begin{array}{l}\text { For NLR } \geq 4 \text { : } \\
\text { HR for death, } \\
2.79(95 \% \mathrm{Cl} \text {, } \\
\text { I.49-5.23) }\end{array}$ & $\begin{array}{l}\text { For } \mathrm{NLR} \geq 5 \text { : } \\
\text { HR for death, } \\
2.07 \text { ( } 95 \% \mathrm{Cl} \text {, } \\
\text { I.3-3.3); HR } \\
\text { for progression, } \\
\text { I.43 (95\% Cl, } \\
\text { I.02-2.00) }\end{array}$ & $\begin{array}{l}\text { For NLR >2.8: } \\
\text { HR for death, } \\
\text { I } 09(95 \% \mathrm{Cl} \text {, } \\
\text { I.04-I.I3; } \\
p=0.0002)\end{array}$ & $\begin{array}{l}\text { For NLR >4.59: } \\
\text { HR for death 2.4I } \\
(95 \% \mathrm{Cl}, \mathrm{I} . \mathrm{I} \mathrm{I}-5.24 \text {; } \\
p=0.027) \text {; HR for } \\
\text { progression, } 2.08 \\
(95 \% \mathrm{Cl}, \mathrm{I} .10-3.95 \text {; } \\
p=0.027 \text { ) }\end{array}$ & $\begin{array}{l}\text { For NLR } \\
\geq 4: \text { HR for } \\
\text { death, } 3.133 \\
\text { ( } p=0002) ; \\
\text { HR for } \\
\text { progression, } \\
1.518(p=0.120)\end{array}$ & $\begin{array}{l}\text { For } N L R \geq 4 \\
\text { or }<4: \text { HR for } \\
\text { death, I.82 } \\
\text { ( } p=023) ; \\
\text { HR for progression, } \\
\text { I.83 ( } p=0.06)\end{array}$ \\
\hline Study design & $\begin{array}{l}\text { Prospective } \\
\text { cohort }\end{array}$ & $\begin{array}{l}\text { Retrospective } \\
\text { cohort }\end{array}$ & $\begin{array}{l}\text { Retrospective } \\
\text { cohort }\end{array}$ & $\begin{array}{l}\text { Prospective } \\
\text { cohort }\end{array}$ & $\begin{array}{l}\text { Retrospective } \\
\text { cohort }\end{array}$ & $\begin{array}{l}\text { Retrospective } \\
\text { cohort }\end{array}$ & $\begin{array}{l}\text { Retrospective } \\
\text { cohort }\end{array}$ \\
\hline
\end{tabular}

Abbreviations: GU, genitourinary; HR, hazard ratio; ICl, immune checkpoint inhibitor; NLR, neutrophil-to-lymphocyte ratio; NSCLC, non-small-cell lung cancer; OS, overall survival; PFS, progression-free survival; RCC, renal cell carcinoma; UC, urothelial carcinoma. 


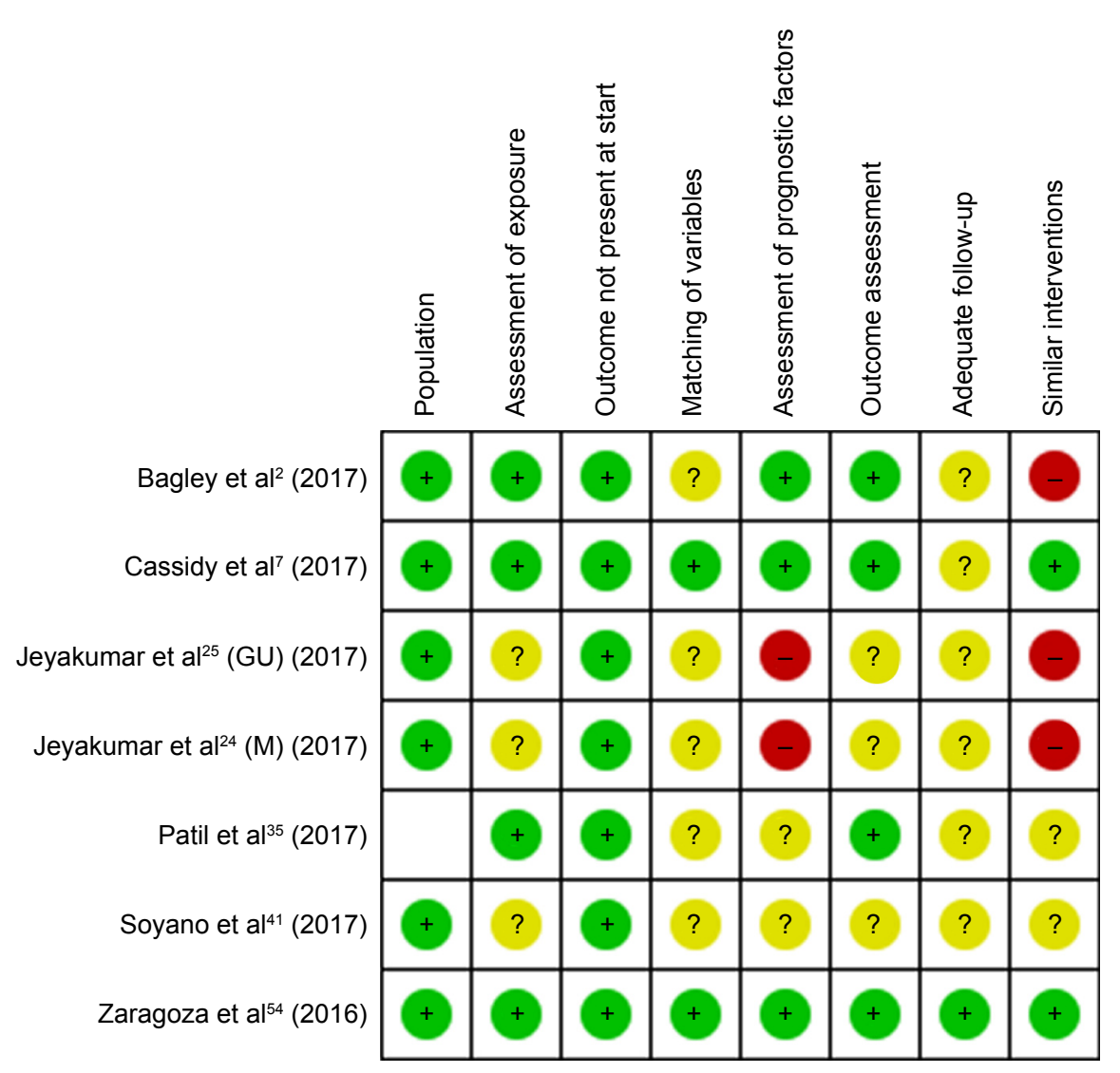

Figure 2 Summary of risk of bias for included studies on prognostic utility of the NLR in patients receiving immune checkpoint inhibitors. Abbreviations: GU, genitourinary; M, melanoma; NLR, neutrophil-to-lymphocyte ratio.

Oncology Group (ECOG) score were reported across studies and assessment of outcomes factored these as well.

\section{Outcomes of included studies}

A high NLR resulted in worse OS (HR, 1.92; 95\% CI, 1.29-2.87; $p=0.001)$ and PFS (HR, 1.66; 95\% CI, 1.38-2.01; $p<0.00001)$. Subgroup analysis across types of malignancy treated with ICI is as follows: melanoma (HR, 2.18; 95\% CI, 1.66-2.84; $p<0.0001)$ and NSCLC (HR, 1.63; 95\% CI, $0.92-2.88 ; p=0.09)$. The single study on GU cancers also showed worse OS (HR, 1.82; 95\% CI, 0.68-4.84; $p=0.23$ ). High NLR also corresponded to worse PFS for melanoma (HR, 1.73; 95\% CI, 1.33-2.25; $p<0.0001)$ and NSCLC (HR, 1.56; 95\% CI, 1.14-2.13; $p=0.005$ ). The single study on GU cancers also showed worse PFS (HR, 1.83; 95\% CI, 0.97-3.44; $p=0.06$ ).

A high NLR also showed worse OS and PFS across all ICIs (OS: HR, 1.92; 95\% CI, 1.29-2.87; $p=0.001$; PFS: HR, $1.66 ; 95 \%$ CI, $1.38-2.01 ; p<0.00001)$. Subgroup analysis by ICI showed similar results: for ipilimumab (OS: HR, 2.05; 95\% CI, 1.54-2.04; $p<0.00001$; PFS: HR, 1.81; 95\% CI,
$1.33-2.45 ; p=0.0002)$ and worse OS for nivolumab (OS: HR, 1.43; 95\% CI, 0.77-2.69; $p=0.26$; PFS: HR, 1.43; 95\% CI, 1.02-2.00; $p=0.04)$. For studies that did not specify ICI used and those that pooled nivolumab and pembrolizumab, OS and PFS showed similar results (OS: HR, 2.52; 95\% CI, 1.58-4.02; $p<0.0001$; PFS: HR, 1.76; 95\% CI, 1.25-2.47; $p=0.001$ ). Figures 3-6 summarize the outcomes of studies in this meta-analysis.

\section{Heterogeneity}

Significant heterogeneity was noted for pooled data on OS because of a single study. Heterogeneity diminished in pooled data on PFS since the same study did not report PFS data and hence was excluded. The $\chi^{2}$ and $I^{2}$ showed that the null hypothesis of homogeneity was rejected as has been observed.

\section{Risk of publication bias}

Due to the retrospective nature of most studies included, the authors cannot determine whether selective reporting was performed in any of the articles reviewed. 


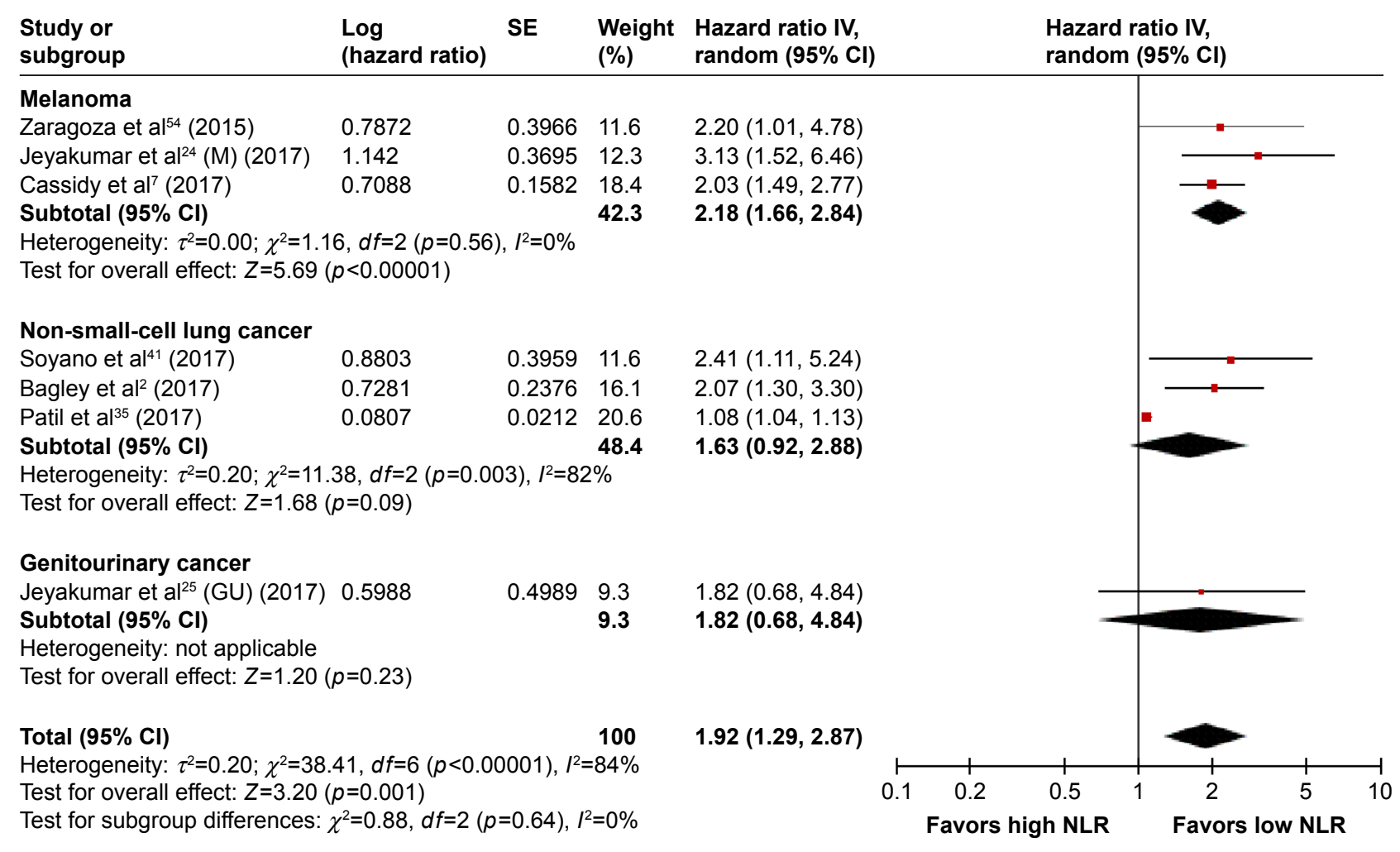

Figure 3 HR for overall survival by NLR according to the tumor type.

Abbreviations: GU, genitourinary; HR, hazard ratio; M, melanoma; NLR, neutrophil-to-lymphocyte ratio; SE, standard error.

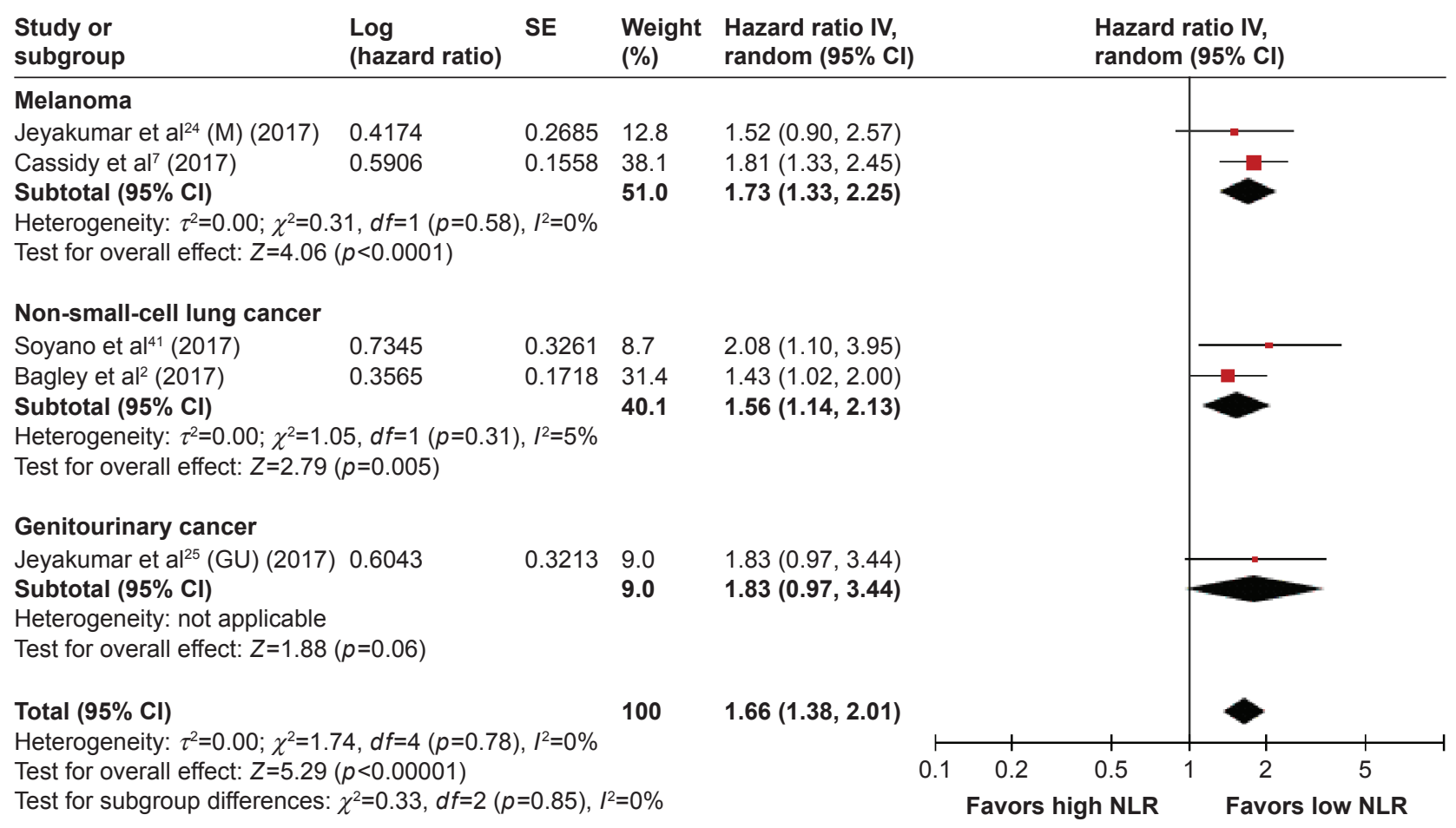

Figure 4 HR for progression-free survival by NLR according to the tumor type.

Abbreviations: GU, genitourinary; HR, hazard ratio; M, melanoma; NLR, neutrophil-to-lymphocyte ratio; SE, standard error. 


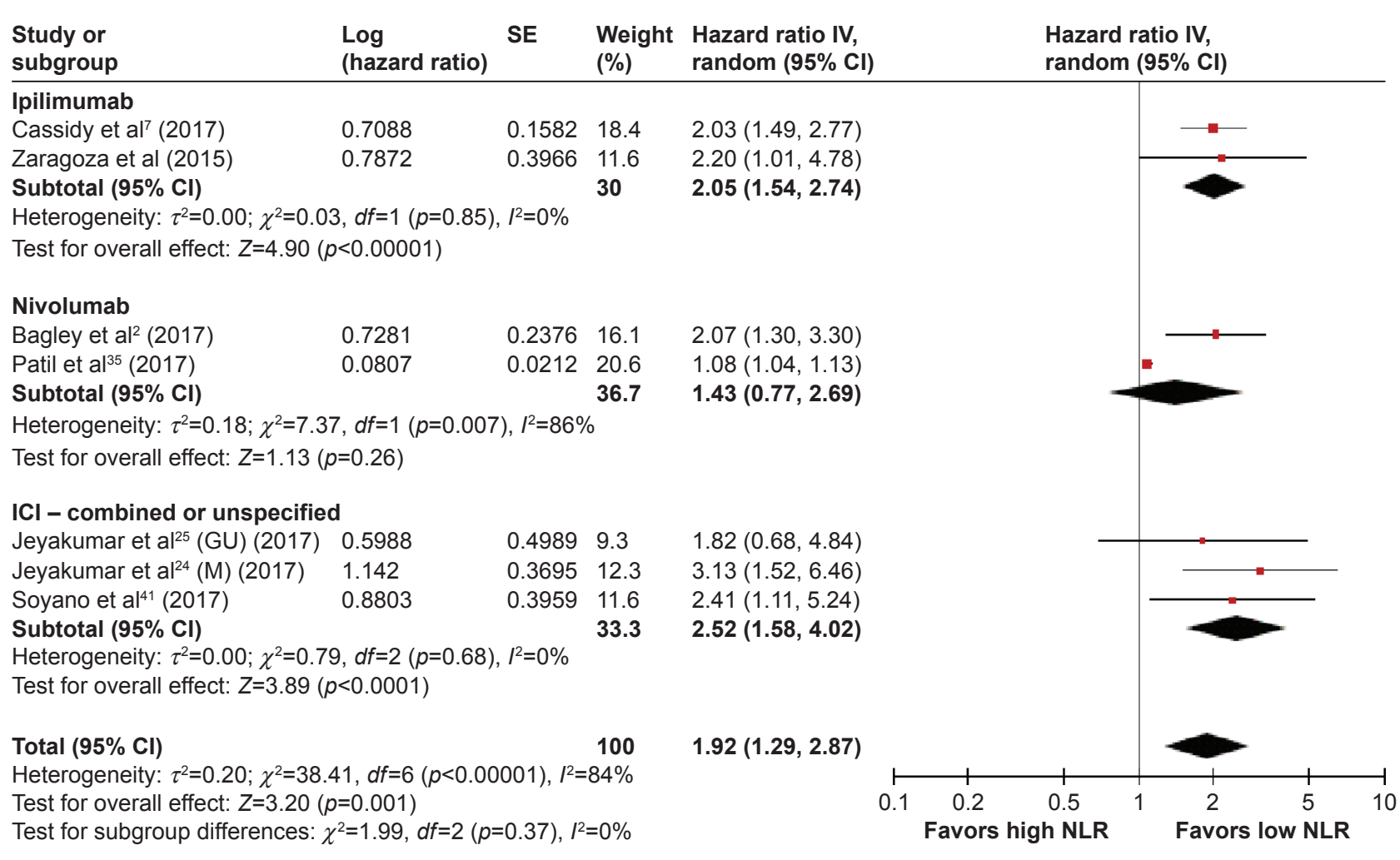

Figure 5 HR for overall survival by NLR according to the immune checkpoint inhibitor.

Abbreviations: GU, genitourinary; HR, hazard ratio; M, melanoma; NLR, neutrophil-to-lymphocyte ratio; SE, standard error.

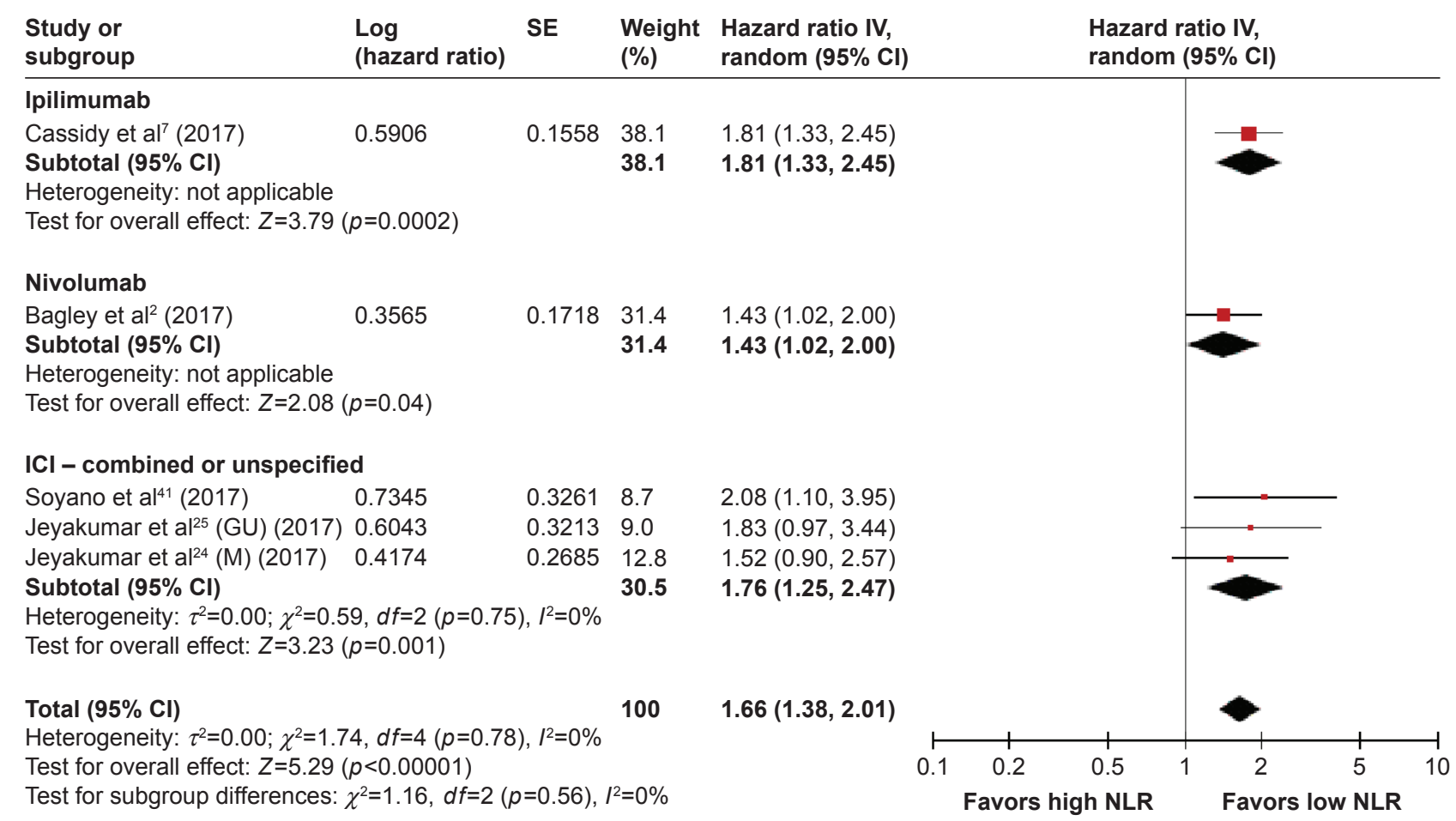

Figure 6 HR for progression-free survival by NLR according to the immune checkpoint inhibitor.

Abbreviations: GU, genitourinary; HR, hazard ratio; M, melanoma; NLR, neutrophil-to-lymphocyte ratio; SE, standard error. 


\section{Discussion}

Inflammation has been seen to play a critical role in tumor development. Specifically, it has been observed to have a detrimental effect on patient survival in cancer. Increasing NLR has been demonstrated to be a harbinger of a more dismal prognosis across different cancers. . $^{2,27,37,49,51-53}$ The utility of NLR lies in its ability to reflect the degree of inflammation present in a patient. . $^{2,24}$

This meta-analysis aims to study the effectiveness of this biomarker in the prediction of prognosis among patients receiving ICI. Although including relatively few studies, this review has shown that across several cancer types, and at least between the ICIs, ipilimumab and nivolumab and based on the pooled data from nivolumab and pembrolizumab, NLR is a potentially useful prognosticating tool. It is particularly advantageous because of its availability and noninvasive nature.

However, NLR is not without its criticisms. To begin, the lack of a uniform cutoff value among studies shows that the understanding of this biomarker is still immature, and this observation has also been made by other authors. ${ }^{14,30}$ Although a high NLR value is clearly detrimental, it is uncertain what value of NLR is ideally used to estimate prognosis across ICI-treated patient groups. In addition, NLR is a dynamic marker, varying across time points during a patient's treatment course. While this review has looked at the prognostic utility of baseline NLR values, other studies have also looked at the change in NLR during treatment as another potential prognostic tool. ${ }^{7,12,26}$ Whether a single NLR determination or several over a time course is better at estimating prognosis in ICI-treated patients has not been established.

By showing that NLR can predict outcomes in ICI-treated patients, this study supports the idea that inflammation, through different mediators, attenuates the effectiveness of immune checkpoint blockade. Furthermore, it presses the need to search for potentially targetable mediators of inflammation in patients treated with ICIs as suggested by other authors. ${ }^{28}$ Targeting these pro-inflammatory molecules may, in turn, directly affect patient outcomes.

We put forward several points for future research focus. First, methods employed in study inclusion may be a source of bias because not all studies on ICI routinely report on NLR data, which impacts the robustness of the analysis performed here. Second, most of the included studies were of retrospective design. Data collected from registries are prone to unintentional (missing data) or even intentional bias (patients being left out of reporting). ${ }^{32}$ Third, the effects of cointerventions (eg, colony-stimulating factors for supportive care) and confounders (eg, timing of treatment) are difficult to account for.

Finally, it is worth noting that the weight of study-level data as used in this analysis may not be the same as that of patient-level data due to the latent effects the respective analyses already applied to them. Nevertheless, the results of this study show that NLR is an effective tool in prognosticating patients treated with ICIs.

\section{Conclusion}

In this meta-analysis, we have shown that a high NLR is associated with poorer outcomes across studies of ICI-treated patients. This shows that NLR has the potential to be a readily available prognostic indicator for patients receiving ICI based on available studies. Further research will allow better understanding of this biomarker and its role in the use of ICIs in cancer.

\section{Areas for further research}

- We recommend that NLR be measured in succeeding therapy trials to aid in future analysis.

- Determination of a standardized cutoff for NLR values is worth investigating.

- Utility of baseline NLR coupled with changes in NLR in a prospective setting to better elucidate the value of NLR in prognosticating outcomes in ICI-treated patients.

\section{Disclosure}

The authors report no conflicts of interest in this work.

\section{References}

1. Badoual C, Hans S, Fridman W, Brasnu D. Revisiting the prognostic value of regulatory T cells in patients with cancer. J Clin Oncol. 2009; 27(19):e5-e6.

2. Bagley SJ, Kothari S, Aggarwal C, et al. Pretreatment neutrophilto-lymphocyte ratio as a marker of outcomes in nivolumab-treated patients with advanced non-small-cell lung cancer. Lung Cancer. 2017 106:1-7.

3. Blank C, Enk A. Therapeutic use of anti-CTLA-4 antibodies. Int Immunol. 2014;27(1):3-10.

4. Bowen RC, Little NAB, Harmer JR, et al. Neutrophil-to-lymphocyte ratio as prognostic indicator in gastrointestinal cancers: a systematic review and meta-analysis. Oncotarget. 2017;8(19):32171-32189.

5. Buchbinder E, Desai A. CTLA-4 and PD-1 pathways similarities, differences, and implications of their inhibition. Am J Clin Oncol. 2016; 39:98-106.

6. Cao J, Zhu X, Zhao X, Li X-F, Xu R. Neutrophil-to-lymphocyte ratio predicts PSA response and prognosis in prostate cancer: a systematic review and meta-analysis. PLoS One. 2016;11(7):e0158770.

7. Cassidy MR, Wolchok RE, Zheng J, et al. Neutrophil to lymphocyte ratio is associated with outcome during ipilimumab treatment. EBioMedicine. 2017;18:56-61.

8. Chen L. Co-inhibitory molecules of the B7-CD28 family in the control of T-cell immunity. Nat Rev Immunol. 2004;4(5):336-347. 
9. Cheng H, Luo G, Lu Y, et al. The combination of systemic inflammation-based marker NLR and circulating regulatory $\mathrm{T}$ cells predicts the prognosis of resectable pancreatic cancer patients. Pancreatology. 2016;16(6):1080-1084.

10. Diaz-Montero C, Salem ML, Nishimura MI, Garrett-Mayer E, Cole DJ, Montero AJ. Increased circulating myeloid-derived suppressor cells correlate with clinical cancer stage, metastatic tumor burden, and doxorubicin-cyclophosphamide chemotherapy. Cancer Immunol Immunother. 2009;58:49-59.

11. Direnzo D, Shah AA, Bingham CO, Cappelli LC [webpage on the Internet]. The immune checkpoint inhibitors unleashed to fight cancer. Available from: http://www.the-rheumatologist.org/article/ immune-checkpoint-inhibitors-unleashed-fight-cancer/. Accessed November 13, 2017.

12. Di Giacomo AM, Calabrò L, Danielli R, et al. Long-term survival and immunological parameters in metastatic melanoma patients who responded to ipilimumab $10 \mathrm{mg} / \mathrm{kg}$ within an expanded access programme. Cancer Immunol Immunother. 2013;62:1021-1028.

13. DerSimonian R, Laird N. Meta-analysis in clinical trials. Control Clin Trials. 1986;7(3):177-188.

14. Faria SS, Fernandes PC Jr, Silva MJ, et al. The neutrophil-to-lymphocyte ratio: a narrative review. Ecancermedicalscience. 2016;10:702.

15. Finotello F, Trajanoski Z. New strategies for cancer immunotherapy: targeting regulatory T cells. Genome Med. 2017;9:10.

16. Flammiger A, Weisbach L, Huland H, et al. High tissue density of FOXP3+ T cells is associated with clinical outcome in prostate cancer. Eur J Cancer. 2013;49:1273-1279.

17. Fouad Y, Aanei C. Revisiting the hallmarks of cancer. Am J Cancer Res. 2017;7(5):1016-1036.

18. Gibney GT, Weiner LM, Atkins MB. Predictive biomarkers for checkpoint inhibitor-based immunotherapy. Lancet Oncol. 2016;16: e542-e551.

19. Gonda K, Shibata M, Kanke Y, et al. Circulating myeloid-derived suppressor cells (MDSC) and correlation to poor prognosis, Th2polarization, inflammation, and nutritional damages in patients with gastric cancer. J Clin Oncol. 2013;31(15 Suppl):3063.

20. Granot Z, Jablonska J. Distinct functions of neutrophil in cancer and its regulation. Mediators Inflamm. 2015;2015:701067.

21. Hanahan D, Weinberg R. Hallmarks of cancer: the next generation. $J$ Cell. 2011;144(5):646-674.

22. Hoos A, Ibrahim R, Korman A, et al. Development of ipilimumab: contribution to a new paradigm for cancer immunotherapy. Semin Oncol. 2010;37(5):533-546.

23. Ipilimumab. USA: Bristol-Myers Squibb; c2017 [cited November 13, 2017]. Available from: https://packageinserts.bms.com/pi/pi_yervoy. pdf. Accessed December 21, 2017.

24. Jeyakumar G, Bumma N, Kim S, et al. Neutrophil lymphocyte ratio (NLR) as a predictor of outcomes with immune checkpoint inhibitor (ICI) therapy in genitourinary cancer and melanoma. J Clin Oncol. 2017;35(Suppl 7S):abstract 37.

25. Jeyakumar G, Bumma N, Kim S, et al. Neutrophil lymphocyte ratio (NLR) as a clinical biomarker predictive of outcomes with immune checkpoint inhibitor therapy in genitourinary cancers. $\mathrm{J}$ Clin Oncol. 2017;35(Suppl 6S):abstract 453.

26. Khoja L, Atenafu EG, Templeton A, et al. The full blood count as a biomarker of outcome and toxicity in ipilimumab-treated cutaneous metastatic melanoma. Cancer Med. 2016;5(10):2792-2799.

27. Kim IY, You SH, Kim YW. Neutrophil-lymphocyte ratio predicts pathologic tumor response and survival after preoperative chemoradiation for rectal cancer. BMC Surg. 2014;14:94.

28. Labomascus S, Fughhi I, Bonomi P, et al. Neutrophil to lymphocyte ratio as predictive of prolonged progression free survival (PFS) and overall survival (OS) in patients with metastatic non-small cell lung cancer (NSCLC) treated with second-line PD-1 immune checkpoint inhibitors. J Clin Oncol. 2017;35(15 Suppl):e14530.

29. Moses K, Brandau S. Human neutrophils: their role in cancer and relation to myeloid-derived suppressor cells. Semin Immunol. 2016;28(2): 187-196.
30. Nivolumab. USA: Bristol-Myers Squibb; c2017 [cited November 13, 2017]. Available from: https://packageinserts.bms.com/pi/pi_opdivo. pdf. Accessed December 21, 2017.

31. Nair V, Elkord E. Immune checkpoint inhibitors in cancer therapy: a focus on T regulatory cells. Immunol Cell Biol. Epub 2017 Sep 3:doi: 10.1111/IMCB.1003.

32. Norvel DC. Study types and bias - don't judge a study by the abstract's conclusion alone. Evid Based Spine Care J. 2010;1(2):7-10.

33. Ohki S, Shibata M, Gonda K, et al. Circulating myeloid-derived suppressor cells are increased and correlate to immune suppression, inflammation and hypoproteinemia in patients with cancer. Oncol Rep. 2012;28(2):453-458.

34. Ohta A. A metabolic immune checkpoint: adenosine in tumor microenvironment. Front Immunol. 2016;7:109.

35. Patil PD, Khunger M, Rakshit S, et al. Pre-treatment hematological markers as a predictive biomarker for survival in patients with nonsmall cell lung cancer treated with nivolumab. J Clin Oncol. 2017; 35(Suppl):abstr11547.

36. Pembrolizumab. USA: Merck; c2017 [cited November 13, 2017 ]. Available from: http://www.merck.com/product/usa/pi_circulars/k/ keytruda/keytruda_pi.pdf. Accessed December 21, 2017.

37. Pine JK, Morris E, Hutchins GG, et al. Systemic neutrophil-tolymphocyte ratio in colorectal cancer: the relationship to patient survival, tumour biology and local lymphocytic response to tumour. Br J Cancer. 2015;113(2):204-211.

38. Sagiv JY, Michaeli J, Assi S, et al. Phenotypic diversity and plasticity in circulating neutrophil subpopulations in cancer. Cell Rep. 2015;10: $562-573$.

39. Salama P, Phillips M, Grieu F, et al. Tumor-infiltrating FOXP3 ${ }^{+}$ $\mathrm{T}$ regulatory cells show strong prognostic significance in colorectal cancer. J Clin Oncol. 2008;27:186-192.

40. Singel K, Segal B. Neutrophils in the tumor microenvironment: trying to heal the wound that cannot heal. Immunol Rev. 2016;273(1): 329-343.

41. Soyano A, Dholaria BR, Marin-Acevedo JA, et al. Baseline peripheral blood biomarkers associated with clinical outcome of advanced lung cancer in patients treated with anti-PD-1 antibody. J Clin Oncol. 2017;35(Suppl):abstre20599.

42. Syed V. TGF-b signaling in cancer. J Cell Biochem. 2016;9999:1-9.

43. Tsai HF, Hsu PN. Cancer immunotherapy by targeting immune checkpoints: mechanism of $\mathrm{T}$ cell dysfunction in cancer immunity and new therapeutic targets. J Biomed Sci. 2017;24:35.

44. Maleki Vareki S, Garrigós C, Duran I. Biomarkers of response to PD-1/PD-L1 inhibition. Crit Rev Oncol Hematol. 2017;116:116-124.

45. Vijayan D, Young A, Teng MWL, et al. Targeting immunosuppressive adenosine in cancer. Nat Rev Cancer. 2017;17(12):709-724.

46. Wang X, Teng F, Kong L, Yu J. PD-L1 expression in human cancers and its association with clinical outcomes. Onco Targets Ther. 2016; 9:5023-5039.

47. Ward-Hartstonge K, Kemp R. Regulatory T-cell heterogeneity and the cancer immune response. Clin Trans Immunol. 2017;6:e154.

48. Whiteside $\mathrm{T}$. The role of regulatory $\mathrm{T}$ cells in cancer immunology. Immunotargets Ther. 2015;4:159-171.

49. Williams KA, Labidi-Galy SI, Terry KL, et al. Prognostic significance and predictors of the neutrophil-to-lymphocyte ratio in ovarian cancer. Gynecol Oncol. 2014;132(3):542-550.

50. Wolchok JD, Hodi FS, Weber JS, et al. Development of ipilimumab: a novel immunotherapeutic approach for the treatment of advanced melanoma. Ann N Y Acad Sci. 2013;1291(1):1-13.

51. Yin X, Xiao Y, Li F, Qi S, Yin Z, Gao J. Prognostic role of neutrophilto-lymphocyte ratio in prostate cancer: a systematic review and metaanalysis. Medicine. 2016;95(3):e2544.

52. Xue T-C, Zhang L, Xie X-Y, et al. Prognostic significance of the neutrophil-to-lymphocyte ratio in primary liver cancer: a meta-analysis. PLoS One. 2014;9(5):e96072.

53. Yin Y, Wang J, Wang X, et al. Prognostic value of the neutrophil to lymphocyte ratio in lung cancer: a meta-analysis. Clinics (Sao Paulo). 2015;70(7):524-530. 
54. Zaragoza J, Caille A, Beneton N, et al. High neutrophil to lymphocyte ratio measured before starting ipilimumab treatment is associated with reduced overall survival in patients with melanoma. $\mathrm{Br} J$ Dermatol. 2016;174:146-151.
55. Zilio S, Serafini P. Neutrophils and granulocytic MDSC: the Janus God of cancer immunotherapy. Vaccines (Basel). 2016;4(3):E31.

\section{Publish your work in this journal}

OncoTargets and Therapy is an international, peer-reviewed, open access journal focusing on the pathological basis of all cancers, potential targets for therapy and treatment protocols employed to improve the management of cancer patients. The journal also focuses on the impact of management programs and new therapeutic agents and protocols on

\section{Dovepress}

patient perspectives such as quality of life, adherence and satisfaction. The manuscript management system is completely online and includes a very quick and fair peer-review system, which is all easy to use. Visit http://www.dovepress.com/testimonials.php to read real quotes from published authors.

Submit your manuscript here: http://www.dovepress.com/oncotargets-and-therapy-journal 